\title{
Contribution to the modelling of a cross-country skier skiing in its environment
}

\author{
Françoise Rey ${ }^{1}$, André Ferrand ${ }^{1}$ and Hejer Makhloufi ${ }^{2, a}$ \\ 1 INSA, UPS, Mines Albi, ISAE, ICA (Institut Clément Ader), Université de Toulouse, 135 avenue de Rangueil, \\ 31077 Toulouse, France \\ 2 CNRS, LAAS, 7 avenue du Colonel Roche, 31077 Toulouse, France
}

Received 29 September 2011, Accepted 10 April 2012

\begin{abstract}
The studied movement is the "offset" skating technique. It is typically used by the skier for higher-force-requirement situations: climbing up a hill, handling conditions (slow snow or head-wind) or in low force situations. It is a thrust with the both poles for each full stroke-cycle of the legs. Firstly, we have made a model of a skier for a movement cycle and secondly a mechanical analysis to validate this model. This work was made thanks to the biomechanics software "LifeModeler", plug-in of ADAMS software, which is a kinematic and dynamic simulation tool. LifeModeler creates a human body as an anthropomorphic robot and ADAMS allows equipping it with tools and placing it on a ground. We have built a dynamic model in 3D for the skier in its environment, that is, with its equipment on the snow. The final goal is to analyze performances parameters effect and to maximize the movement effectiveness, what is possible by perfecting gesture, equipment and by optimizing materials used in this sport.
\end{abstract}

Key words: Modelling / skating / dynamics / biomechanics / kinematics

Résumé - Contribution à la modelisation d'une skieuse de fond dans son environnement. Le mouvement réalisé par la skieuse est «le décalé », il est souvent utilisé en skating lors des montées. Il correspond à une poussée décalée des bras associée à une poussée latérale de chaque jambe. Dans un premier temps, une modélisation du corps humain de la skieuse réalisant un cycle de décalé et une analyse mécanique, validant la modélisation, ont été effectuées. Ce travail est réalisé grâce à un logiciel de biomécanique « LifeModeler », module du logiciel ADAMS, logiciel de modélisation et de simulation cinématique et dynamique. LifeModeler permet de modéliser le corps humain comme un robot anthropomorphe et ADAMS permet de l'équiper sur un sol. L'étape suivante a été de construire un modèle dynamique en 3D de la skieuse dans son environnement, c'est-à-dire, avec son matériel sur la neige. Le but final est d'analyser l'effet de certains paramètres sur les performances et de rendre le geste le plus efficace possible, soit en l'améliorant au niveau moteur, soit en perfectionnant le matériel et en optimisant les matériaux utilisés dans ce sport.

Mots clés : Modélisation / skating / dynamique / biomécanique / cinématique

\section{Introduction}

The purpose of this paper is to present the methods used to create the environment and its integration in a model of skier. We have done a first mechanical analysis of this sport gesture in [1] by using a kinematic and dynamic modelling of the skier. The software LifeModeler [2], which is a plug-in of the Adams software [3], was used to create this human body model. In the goal to obtain a more realistic behavior, we create a snowy ground and an equipment (skis and poles) by using ADAMS

\footnotetext{
${ }^{a}$ Corresponding author: hmakhlou@laas.fr
}

software, and then we merge the equipment into the human model.

We present the various stages succession for the building of our recent model. First of all, we describe the steps of modeling and the difficulties of the environment design: snowy ground and specific equipment. Then, we show the connections complexity between the skier's body and its equipment and also the complexity of the contact between this equipment and the snow during the movement. The outcome of our work is in the kinematic and dynamic modelling of the skier in its environment which no-one have done yet. A qualitative analysis allows checking the validity of the model. 

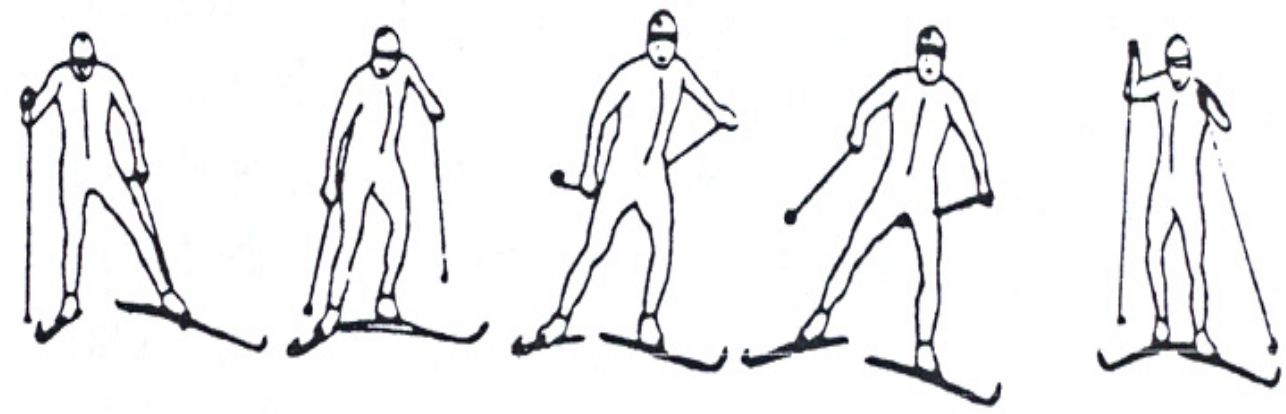

Fig. 1. Chronophotography of the offset technique, Bilodeau et al. [4].

\section{Nomenclature}

\begin{tabular}{|c|c|}
\hline$C_{\mathrm{d}}$ & friction dynamic coefficient \\
\hline$C_{\max }$ & $\begin{array}{l}\text { maximum value of the damping coefficient } \\
C_{\mathrm{t}}\left(\mathrm{N} \text { s.mm }{ }^{-1}\right)\end{array}$ \\
\hline$C_{\mathrm{s}}$ & friction static coefficient \\
\hline $\mathrm{C}_{\mathrm{t}}$ & damping coefficient $\left(\mathrm{N} \mathrm{s} \mathrm{mm}^{-1}\right)$ \\
\hline$F$ & contact force $(\mathrm{N})$ \\
\hline$K$ & stiffness $\left(\right.$ N.mm $\left.{ }^{-1}\right)$ \\
\hline$n$ & $\begin{array}{l}\text { penalty coefficient which has been } \\
\text { experimentally chosen }\end{array}$ \\
\hline$x$ & penetration depth $(\mathrm{mm})$ \\
\hline
\end{tabular}

\section{Definition and analysis of the offset technique}

Offset technique is characterized by Bilodeau [4] into different phases (Fig. 1): a contact phase (ski in touch with the snow) and an air phase. The contact phase is divided in two sub-phases: the sliding one and the propulsion one. The end of the ski propulsion phase and the beginning of the gliding phase are in the same time and correspond to transferring the weight of the upper body from one ski to the other one.

Actions of legs are different; Smith [5] distinguishes "a side leg-strong and a side leg-weak", Coulmy [6] "a side of attack" corresponding to the thrust with the concomitant leg in the thrust of both arms and "a controlateral side".

The movement trajectories of particular body points, the duration of cycle phases and the contact forces may be measured directly.

Several methods of kinematic and dynamics analyses exist:

- force measurement: an array of force sensors is placed beneath a snow surface or a portable force plate is attached to the ski [7]. So, placement of the plate between the ski and binding allowed for measurement of normal forces to the ski surface and measurement of the instantaneous center of pressure of the force [5];

- video analysis : Smith [8-10], Street [11], Gregory [12], Ruby [13], Coulmy [6], carry out a kinematic two or three dimensional analyses;

- combination video analysis and simulation; threedimensional view analysis systems are used by filming a moving person. Markers are attached to the skier in characteristic points. The subject is filmed, and the characteristic points are synchronically transmitted to a computer. Several representations of the offset skating technique exist: a stick representation (Ruby) and a 3D graphical manikin (Verriest [14], Tavernier et al. [15]).

\section{Material and modelling method}

\subsection{Material}

\section{Softwares}

The software LifeModeler [2], was used for the final model wich is a skier (bones and muscles) skiing in its environment. First, we used for human body model. We create snowy ground and equipment, skis and poles, with the software ADAMS [3].

Then, merge the equipment model into human model on LifeModeler.

\section{Subject}

The skier is a 24 years old woman, measuring $166 \mathrm{~cm}$ and weighing $60 \mathrm{~kg}$. She skis on uphill ground (6 degrees) during an international race.

Video analysis

The Ruby's data [13], stemmed from an analysis of a 3D image by geodesic method and filmed with three cameras in a competition, allowed us to collect the Cartesian coordinates of 26 characteristic points situated on the skier and his equipment. This was necessary for the modelling of a human body and equipment during the movement.

Komi [7] and Smith's [5] recorded the contact forces thanks to piezoelectric sensors. This allowed us to validate dynamic models. 


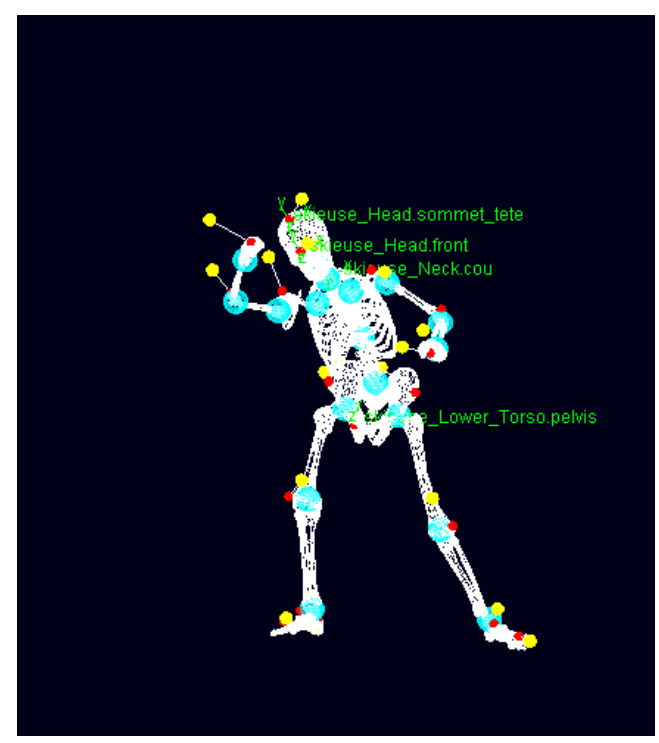

Fig. 2. Equilibrium analysis. Red point is rigidly attached to the bone. Yellow point is a mass less part driven using trajectory data. One spring attaches the two points.

\subsection{Modelling method (Tab. 1)}

LifeModeler's anthropomorphic database automatically creates the bones, joints and muscles of subject. Digitised motion data from a motion capture source (Ruby [13]), is imported into LifeModeler software. "Motion agents" are created at the locations of markers specified. Then, a static analysis is carried out (Fig. 2).

During inverse dynamics simulation, motion agents are associated to the markers of the human model to move according to the prescribed data trajectories and to influence the model via the spring elements. During forward dynamics simulation, with the joint motions recorded from the inverse dynamics simulation, it may be used in a proportional-derivative controller to produce torque to create the motion history. The model is guided by the internal forces (joint torques) and influenced by the external forces (gravity, contact, etc.).

\section{Environment modelling difficulties}

The current skating equipment has undergone a significant evolution over the years. It became light, flexible, more suited to movement with boots, bindings, skis, wax, wrist-straps and poles which are different from those used during the birth of this sport. This equipment was indeed identical to that used in the classic style while body movements are different.

During the modelling work, we tried to take into account this evolution, so that the model can be the more realistic as possible and answer to a performance research.

The environment's model was made on ADAMS software.
Table 1. Building procedure of the skating model.

\begin{tabular}{l}
\hline Human model \\
\hline Generating complete body from anthropometrics \\
database \\
Generating joint set \\
Generating muscle groups \\
Running of the equilibrium simulation \\
\hline Environment \\
\hline Positioning human model in the physical environment \\
Adjusting body starting posture \\
Generating contact reaction forces between body \\
segments and environment or objects \\
\hline Inverse dynamics simulation \\
\hline Import of motion data \\
Adding the environment \\
Driving of the model by the motion agents \\
Running simulation to capture joint angles and muscle \\
stretch \\
\hline Forward dynamics simulation \\
\hline Import joints and muscle forces to include angulation and \\
stretch histories \\
Running simulation with active model interacting with \\
the environment \\
\hline Validation \\
\hline Import test data \\
Compare model motion and environmental reactions \\
\hline
\end{tabular}

\subsection{The pole's model}

To be closer to reality, we wanted to obtain a flexible pole model like it was made in carbon material. The poles are not only stressed in compression, but also in bending because of the complex connections of their extremities: its basket, which penetrates into the snow in one extremity and the action of the hand by the wrist-strap at the other one.

Thus, we built a pole constituted in three parts so that it can be close to the carbon properties. A grip and a rigid basket were associated with a central part made with flexible beam.

The pole was dynamically satisfactory. However, during the movement, the modelling constituted by these three parts moved in an anarchy way. Each part reacts independently of the others. Preferring to focus us on the wrist-strap behavior and on the connection between the hand and the pole, we have chosen a completely rigid simplified pole model.

\subsection{The ski's model}

The flexibility of skating ski is necessary for the technique of the gesture. It involves a weight transfer onto one leg and then to the other one. For this requirement, it was necessary to model a flexible and solid ski. So we created the whole ski with flexible elements. But under the effect of strengths, these elements the too much deformed and penetrated into each other.

We finally opted for an association of rigid and flexible parts. We made a test based on the behaviour of a ski 


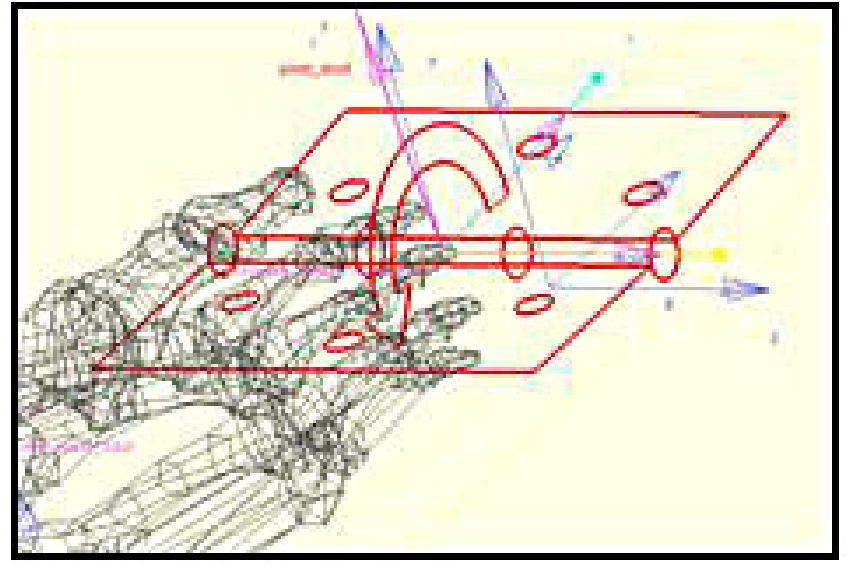

Fig. 3. Localization of the pivot connection at the tiptoe.

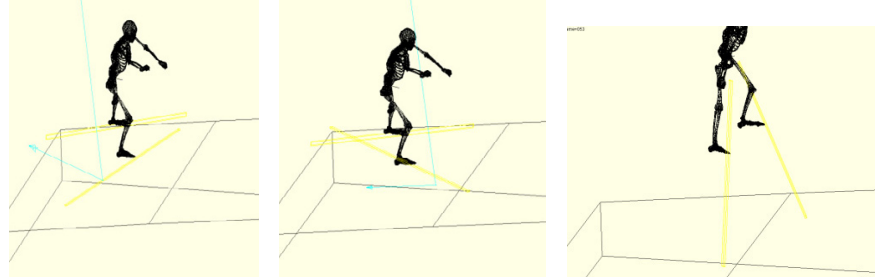

Fig. 4. Ski behavior by using a pivot connection.

submitted to a charge for checking the values of the ski flexibility. This is a very simplified conception but it gives correct dynamic results for the whole model.

\subsection{The binding's model}

The binding has to maintain the foot in the ski's axes. But it also has to free the heel to make possible the movement. It evolved with time: one axis or two axes according to the manufacturer's model. At this time, the trend is a return in a one axis only, the movement of the foot and the ankle allowing an effective glide.

\subsubsection{Development of a pivot connection}

The first attempt corresponds to a simple binding in an axis. By a pivot connection, it reflects the technology of the equipment. However, during the movement, its action can't reflect the gesture of the foot which, by the way of the ankle joint, maintains the ski onto the boot. The pivot connection is modeled by the software, thanks to a position and an axis: the tiptoe is connected to the ski by a horizontal axis (Fig. 3).

The result is not entirely satisfactory. This link gives too much freedom to the ski which touches on the ground several times by the heel and the spatula. Furthermore, the rigidity of skis gives a propulsive effect to the skier who loses the contact with the ground. Then the skis begin to turn $360^{\circ}$ around the foot under the influence of the gravity (Fig. 4).

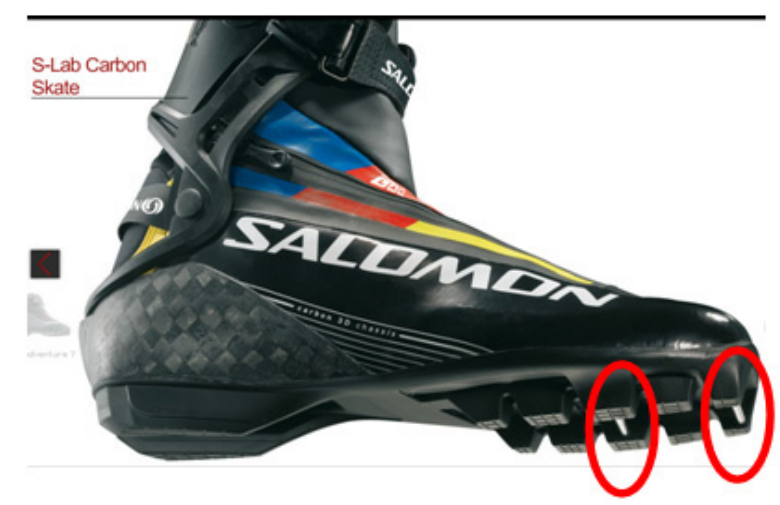

Fig. 5. Skating boot with two axes.

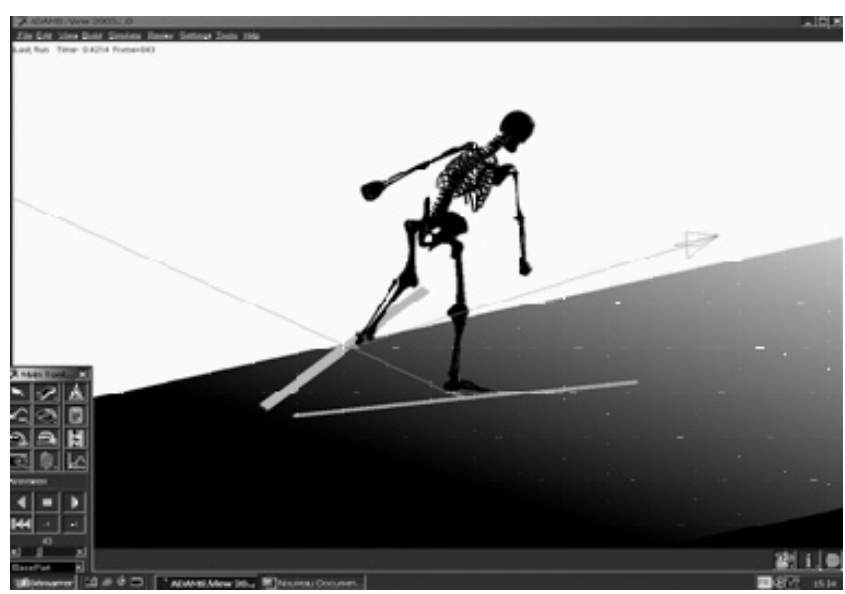

Fig. 6. Spring added between the heel and the ski.

We added a moment of friction on the pivot connection, but it didn't change its behavior, the ski continued to rise.

\subsubsection{Development of a link with two axes: pivot connection} with a spring recall

This design is closer to the binding behavior, corresponding to the commercial "pilot ski binding" launched by the manufacturer Salomon (Fig. 5). On the ski, a special deformable connection makes possible the movement and restitutes a good feeling.

So that the ski can remain closer to the foot and follows its axis, we added a spring between the heel and the ski simulating the strength which recalls the heel towards the ski (Fig. 6).

The tuning of the spring stiffness should allow us to reach a corresponding behavior. At the beginning of the simulation, the ski motion seems correct as long as the heel is going up. But during the way down, it becomes unstable because of increasing strengths applied by the spring. We tried to fit the stiffness of the spring but that didn't give any exploitable results. 
Table 2. Dimensions of the ski pole.

\begin{tabular}{cccc}
\hline & & \multicolumn{2}{c}{ Dimensions (mm) } \\
\hline \multirow{2}{*}{ Grip } & Cylinder 1 & Diameter & Length \\
& Cylinder 2 & 14 & 50 \\
& Cylinder 3 & 10 & 50 \\
\hline Central part & Cylinder 4 & 60 & 1315 \\
\hline Basket & Cylinder 5 & 10 & 40 \\
\hline Extremity part & & &
\end{tabular}

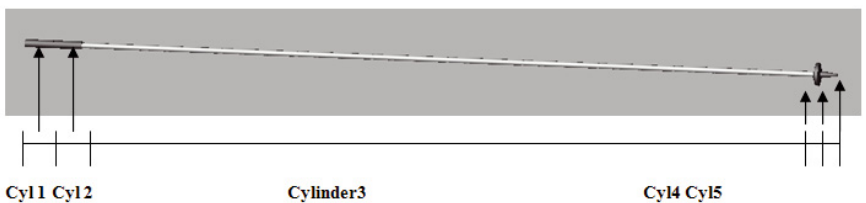

Fig. 7. Model of the ski pole.

\subsubsection{Modelling the binding thanks to a bushing element}

The instability of the ski occurred with a spring alone is easier to control with a bushing connection because this one allows to associate a spring and a shock absorber between two solids in six space directions.

\section{Model of the environment}

\subsection{The pole's model}

A grip modelling is carried out in the aim to merge the pole with the human model. The pole must be controlled by the point which will be in contact with the hand. We create a grip divided into two cylinders, in between a global marker. This marker, associated with a marker located between the inch and the index, will enable us to create a link between the hand and the wriststrap.

We simplified the shape of the basket by modelling it by a disk. The pole is thus constituted by five cylinders (Fig. 7). Their dimensions depend on the skier's size $(166 \mathrm{~cm})$ and are presented in the Table 2.

\subsection{The ski's model}

The ski is constituted by seven elements: three rigid parts, tail, binding, spatula; four flexible beams, two rear parts and two front parts (Fig. 8) (Tab. 3).

Properties of the flexible link are: the connection type at the fasteners points (flexible, rigid or free), the stiffness and damping coefficients. It was, therefore, necessary to discretize the ski in a sufficient number of parts, seven exactly, to obtain a correct ski behavior. A finer discretization would bring more precision but that would increase the computational time. Ski modelling is not symmetrical; the binding is not exactly at the centre but a little bit to the front as it is in the reality.

The ski must have a correct flexibility and must not vibrate under the stress to which it is usually submitted.
By experiments, we found the damping ratio and the camber of the ski according to the weight of the skier $(600 \mathrm{~N})$. We found a deflection of $37 \mathrm{~mm}$ of the higher point and we adjusted the damping ratio on the software between 0 and 1. We simulated the fall of the ski on the ground. The vibrations creating by this fall must be quickly stopped. Different tests with tuning stiffness and damping allowed us to obtain a correct behavior of the ski.

The best values of the mechanical parameters are summarized in the Table 4.

\subsection{The snowy ground's model}

The composition of the snow is a very important parameter for skating and determines the type of ski and wax that the skier will want to use. We have chosen to transcribe the connection between ski and snow by the modelling of the contacts so that they simulate as much as possible the gliding. As for the ground, it is represented by a rigid block. The slope of the uphill is $6^{\circ}$, it respects the data of Ruby [13] (Fig. 9).

After having adjusted the parameters of the skis, we positioned these on the block and we generated the contact.

\subsection{The contacts' model}

The modelling of the interface (ski/snow) is complex because it is conditioned by several parameters which can vary. When gliding, the roughness of the ski and the wax create little water droplets thanks to the heating of the snow (Fig. 10). The specialists try to optimize this aqueous film according to the snow characteristics.

The friction coefficient is essential in this interface; it depends on the speed of the skier, on the roughness of the ski, but also on the snow and waxing characteristic.

Shimbo's works [16] allowed us to find values of the necessary parameters for the modelling of contacts. Indeed, the graph (Fig. 11) shows the friction coefficient evolution of the ski according to the skier's speed. It gives us the static coefficient, the dynamic friction and the transition speed between adherence and dynamic friction.

The second graph (Fig. 12) shows the evolution of the friction dynamic coefficient according to the roughness of a ski sole.

We find in scientific literature average values corresponding to the specificity of skating which is practiced on groomed snow. The parameters of the friction coefficient and of the ski penetration depth in the snow are retained. We also choose a temperature of the snow close to $10^{\circ}$ corresponding to a compact and cold snow as well as an average layer of wax $(0.5 \mathrm{~mm})$. These values are listed in Table 5.

ADAMS proposes many types of contacts such as "Solid-Solid", "Curve-Curve", "Point-Point", "PointPlan", "Curve-Plan", "Sphere-Plan", and "SphereSphere". 
Table 3. Dimensions and weight of the ski.

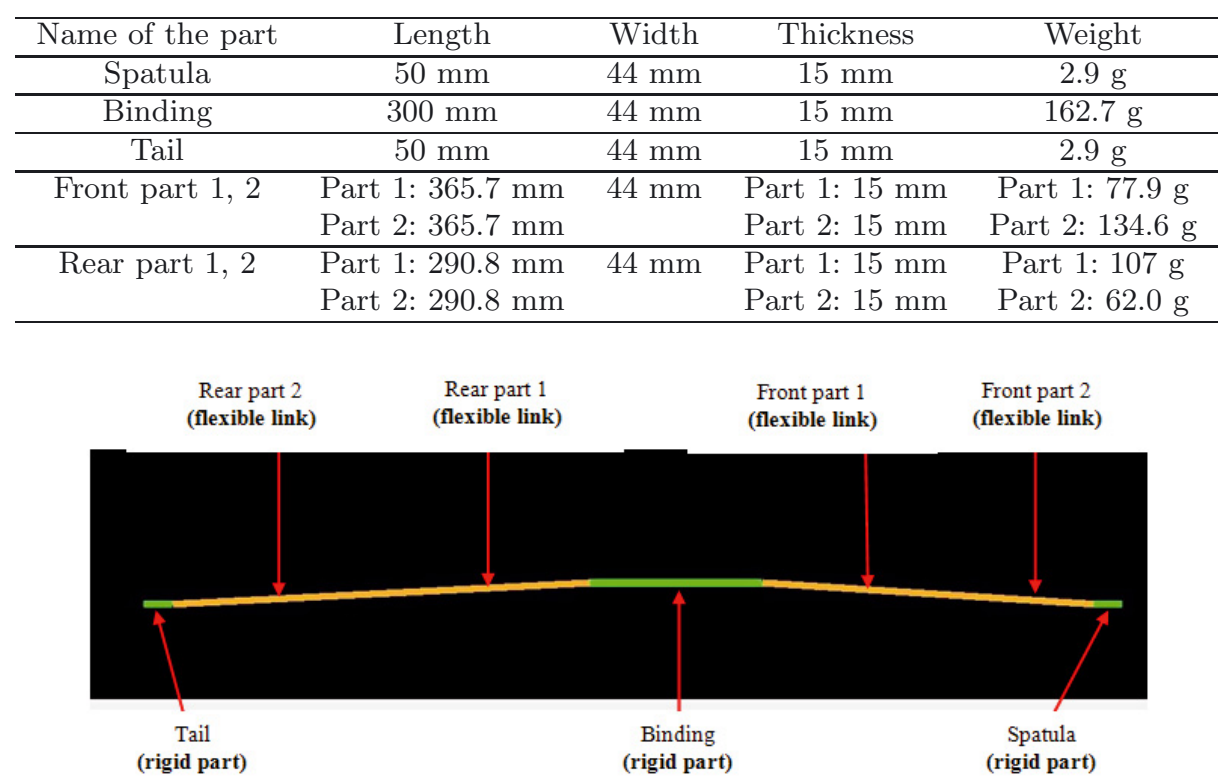

Fig. 8. The ski's model.

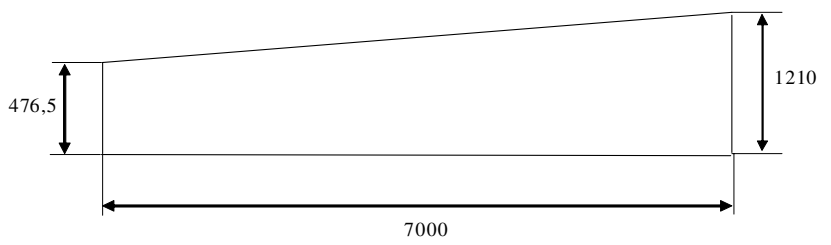

Fig. 9. The snow block dimensions (mm).
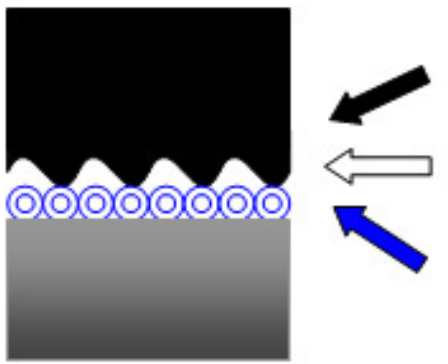

\section{Roughness}

\section{Wax}

Droplets of water

Fig. 10. The gliding of the ski on the snow.

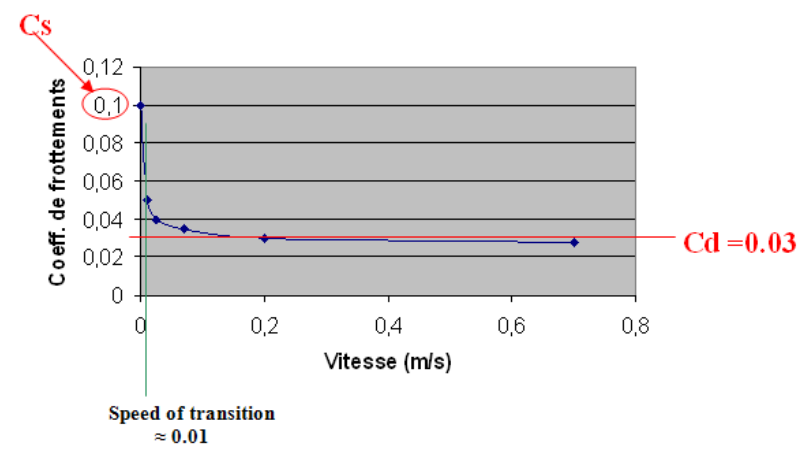

Fig. 11. Friction coefficient according to the speed of the ski on wet and compact snow, Shimbo [16].
Table 4. Ski characteristics.

\begin{tabular}{ccc}
\hline Parameters & Value & Units \\
\hline Damping ratio & 0.3 & $/$ \\
Density & 440 & $\mathrm{~kg} \cdot \mathrm{m}^{-3}$ \\
Thickness of the ski & 15 & $\mathrm{~mm}$ \\
\hline
\end{tabular}

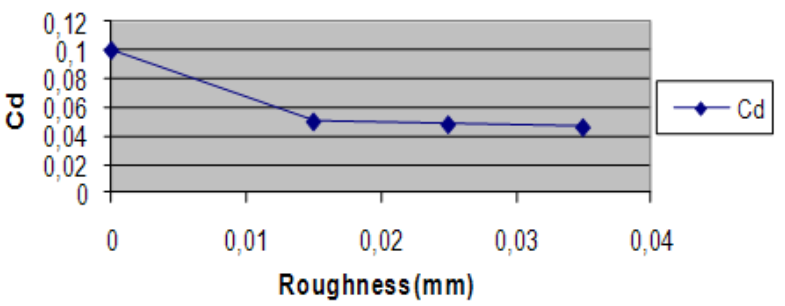

Fig. 12. Evolution of the dynamic friction coefficient according to the ski sole roughness, Shimbo [16].

Table 5. Parameters values for ski/snow contact.

\begin{tabular}{cc}
\hline Name & Parameter values \\
\hline Stiffness $(\mathrm{K})$ & $25.11 \mathrm{~N} . \mathrm{mm}^{-1}$ \\
Penalty coefficient $(n)$ & 1.3 \\
Damping $\left(C_{\mathrm{t}}\right)$ & $1 \mathrm{Ns} . \mathrm{mm}^{-1}$ \\
Penetration depth $(x)$ & $10 \mathrm{~mm}$ \\
Static coefficient & 0.1 \\
Dynamic coefficient & 0.03 \\
Stiction transition & $10 \mathrm{~mm} . \mathrm{s}^{-1}$ \\
Friction transition & $11 \mathrm{~mm} . \mathrm{s}^{-1}$ \\
\hline
\end{tabular}

After taking into account initial conditions of the ski and snow characteristics, a "Solid-Solid" contact is chosen with the "impact" option (Fig. 13). This option enables two parts to have a contact between them, to keep this contact or to remove it at any moment. Contact 


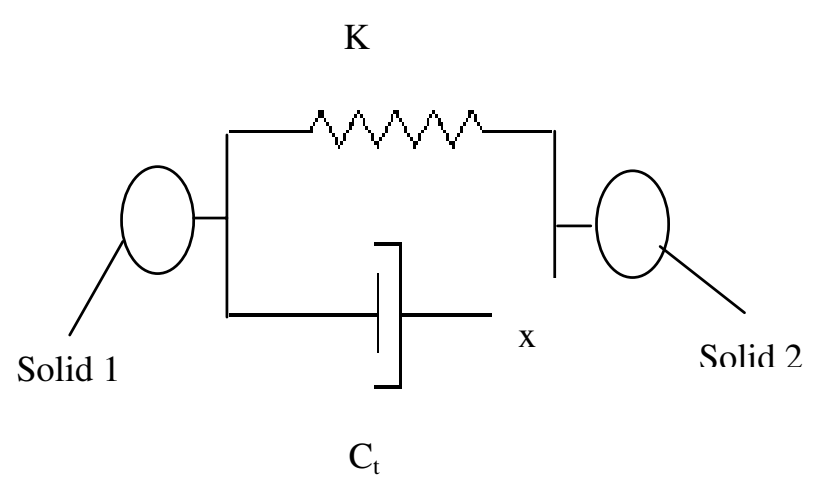

Fig. 13. Contact system between two solids.

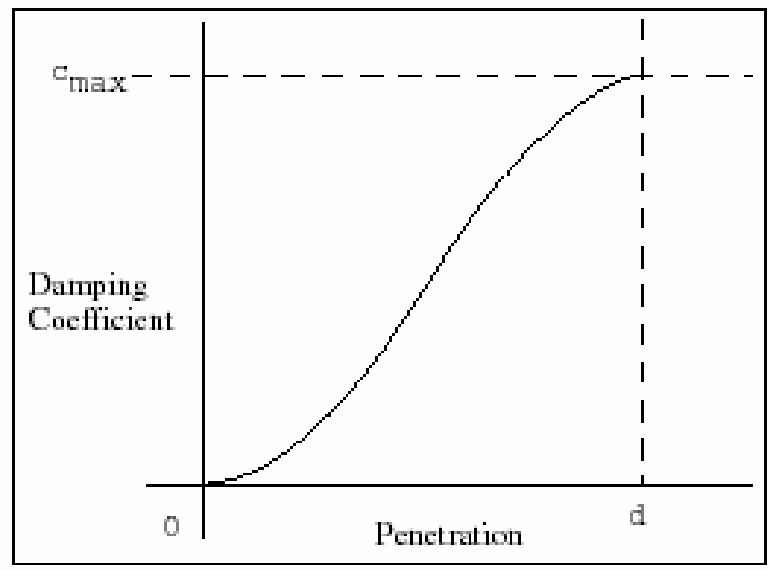

Fig. 14. Damping coefficient versus penetration.

parameters are used to regulate the force intensity with which parts repel each other. The contact force can be represented using the following Equation (1):

$$
F=K x^{n}+C_{\mathrm{t}} \mathrm{d} x / \mathrm{d} t
$$

The force has two components: a spring or stiffness component $\mathrm{K}$ and a damping or viscous component $C_{\mathrm{t}}$. The stiffness component creates a force proportional to " $K$ " and is a function of the distance between two markers, it acts against the penetration. The damping is a function of the penetration speed; it acts against the relative motion. To prevent a discontinuity in the damping force at the contact instant, the damping coefficient is a cubic step function of the penetration. At zero penetration, the damping coefficient is zero. The damping coefficient achieves a maximum, $C_{\max }$, at a penetration defined by the user (Fig. 14),

In the case of a well-waxed polyethylene ski sole, the friction coefficients are very small, about 0.02 to 0.05 during the gliding phase.

Conditions applied to the parts in contact are characterized on the software by a static coefficient $\left(C_{\mathrm{s}}\right)$ and a dynamic coefficient $\left(C_{\mathrm{d}}\right)$ of friction.

By adjustment procedure, we found preferential parameters values for the stiffness, the damping and the exponent $n$.

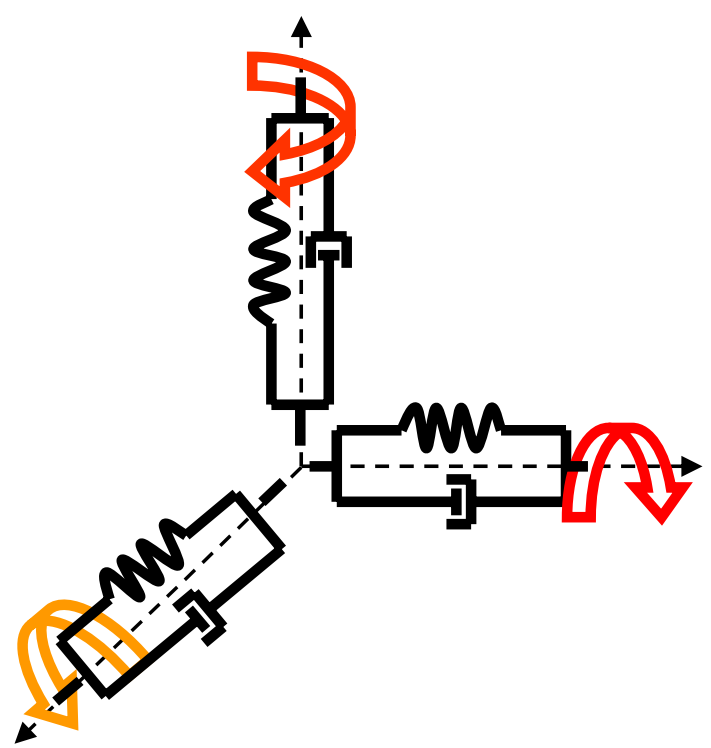

Fig. 15. Simplification of bushing connection.

The exponent $\mathrm{n}$ corresponds to a "penalty coefficient" which characterizes the penetration force. Compared to a spring alone, the contact becomes rigid if $\mathrm{n}$ is higher than 1 , and remains soft if $\mathrm{n}$ ranges from 0 to 1 . For a rigid ground, a value of 1.5 or more is necessary.

The contact and friction conditions are assigned to the seven portions constituting the ski sole. The contact is modelled between the snow block and each portion. For the contacts calculation, the snow marker is the origin of the global marker.

\section{Integration of the environment in the human model}

The physical environment, imported by the way of an ADAMS' file towards the LifeModeler's file, is positioned in the database of the human model. The bushing element is preferred to the traditional mechanical connection standard pivot or ball-joint because the complete restraint of motions can create instabilities and even divergences during simulation. The bushing is a six degree-of-freedom mechanical joint. Each degree is limited by a spring and a dashpot. Stiffness and damping coefficients must be adjusted to obtain the good behavior. This force is applied between markers belonging at each object/body. The force depends on the relative displacement and on the relative velocity of the two markers. In the bushing joint, we have to adjust six springs (3 translational and 3 rotational) and six dashpots (3 translational and 3 rotational) (Fig. 15).

\subsection{Hand bushing}

The complexity of this connection is the specific hand movement. The wrist-strap (Fig. 12) helps to prevent the 

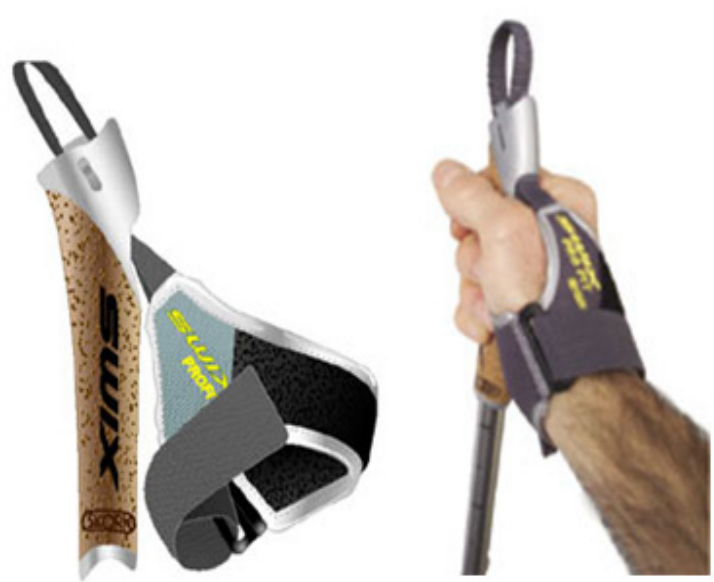

Fig. 16. Skating pole and location of the hand in the wriststrap.

skier from dropping the pole, in the air phase during the return of the arm and also assists with thrusting in poling technique. A marker is created between the inch and the index (between thumb and forefinger); it is associated to the marker of the pole grip centre. After numerous tries, the values of stiffness and damping have been found; they are identical for each hand.

\subsection{Foot bushing}

We created a marker on the big toe. It is associated with the marker of the binding but in reality it's on the first link of the binding with the skating boot. The stiffness and damping values are found identical for each two feet. We can simulate the various binding types thanks to the bushing specificity. It restricts sagittal, transverse and frontal rotations (Fig. 18).

\section{Results}

The inverse dynamic simulation activates the model with the motion agents. With the forward dynamic simulation, the model of skier isn't driven but acts itself on snow.

Many kinematic functions are possible: video, view from any angle, stop simulation, chronophotography, ... But, during the modelling of the skier in its environment, our objective was to get a correct dynamic model to be able to analyze it mechanically. Even if kinematics remains still imperfect, we preferred a model which approximates the more as possible dynamic reality.

After the dynamic simulation, the skier reacts in an autonomous way with her environment; she is not controlled more kinematically. Her weight is taken into account as well as the contact forces. The quality of the model can then be judged.

In fact the results emanating from the forward dynamics simulation will allow a realistic dynamics analysis

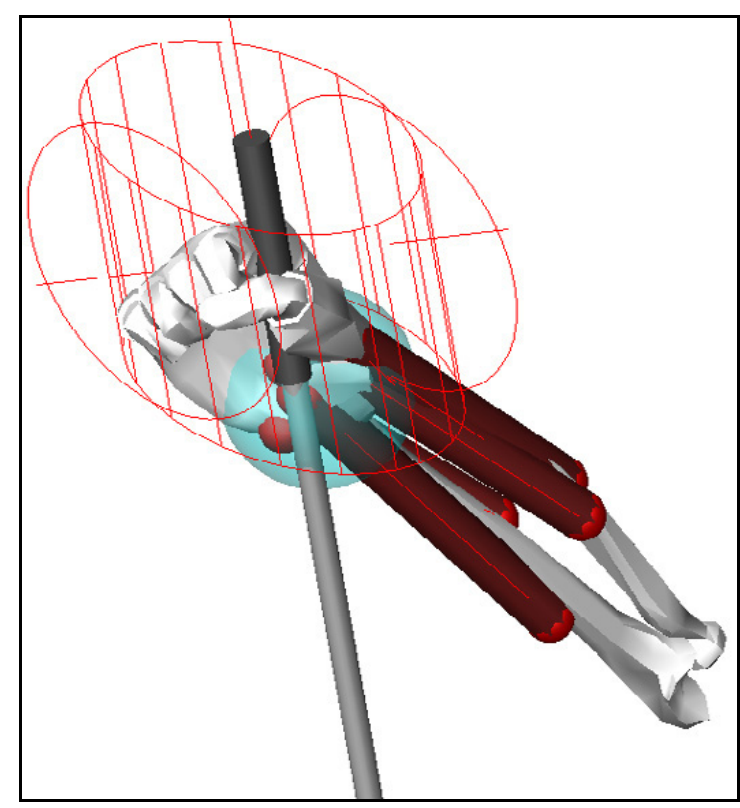

Fig. 17. Visualization of the left profile of the right hand bushing.

because it is the model of the skier with her equipment which acts on snow, without being guided. The contact forces values can be obtained all along the cycle of skier (Fig. 19).

An interesting result of Smith's works [5], on the biomechanical analysis of the cross-country skiing techniques and particularly on the evolution of the skis and poles contact force during a complete cycle of the offset is given (Fig. 20). In our dynamic results (Fig. 21): the poling forces are illustrated by the dotted lines (little dotted-left pole force, big dotted-right pole force); the weak ski force (left ski) is illustrated by the central vertex. Our results compared with Smith's results, represent a similar profile of the curves, except oscillations much more important, at the beginning and the end of the cycle. In our model, the contact force of a ski results from the sum of the contact forces of the 7 parts of the ski.

The contact force of the strong ski, right ski, is illustrated by a curve divided into two on the graphs. The contact force of the weak ski, left ski, increases during a first time, decreases and increases during a last time creating two nearly identical peaks. The values of two graphs are in the same order:the maximum median values reached by the curves are for the strong ski $1300 \mathrm{~N}$ and for the weak ski $900 \mathrm{~N}$. The plant time of pole, order and duration are correct, first the weak pole after the strong. The curves comprise many oscillations, the weak poling force is on average $350 \mathrm{~N}$, with peaks to $800 \mathrm{~N}$ and the strong poling force is on average $400 \mathrm{~N}$ with peaks to $600 \mathrm{~N}$

Thanks to the graph of the poling forces and the skis forces of our model, we can analyse the phases of the cycle (Fig. 22) and compare the percentage from the literature.

The comparison between the percentages of cycle phases of the model and those of other studies 


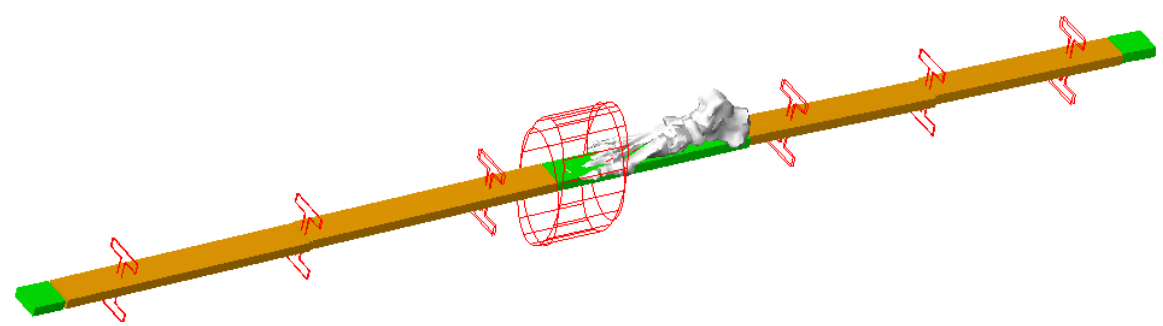

Fig. 18. Visualization of the left profile of the right foot bushing.

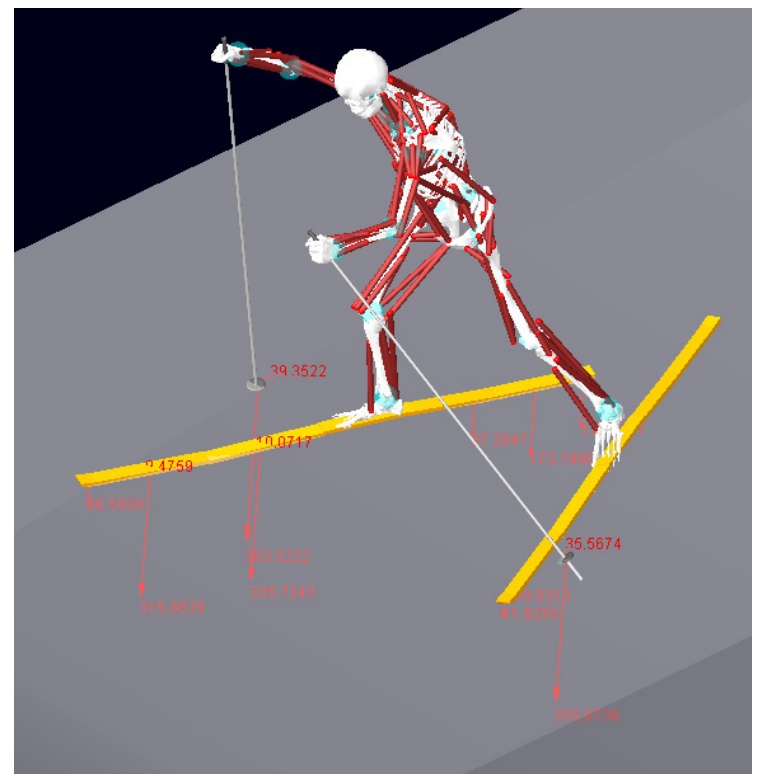

Fig. 19. Sight of $3 / 4$ of the dynamic simulation, contact forces exerted by the skier are visualized by the red arrows.

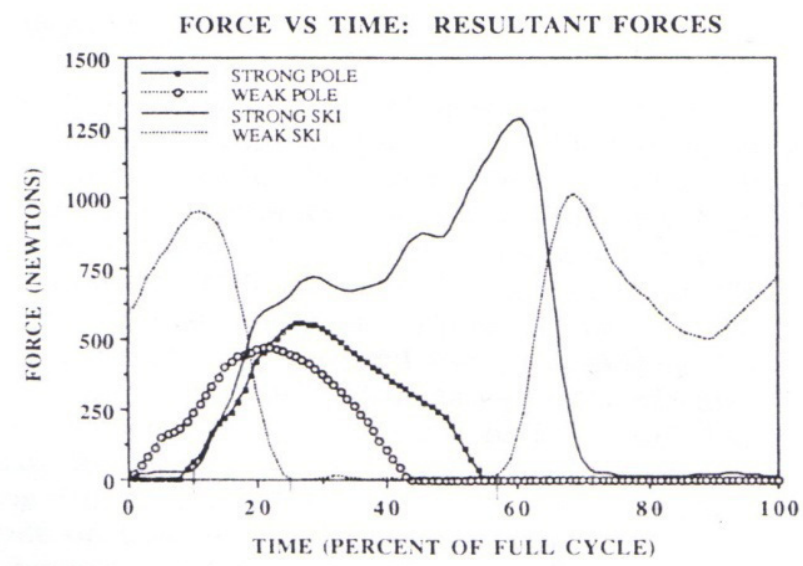

Fig. 20. Poles and skis contact forces during offset, Smith [5].

Bilodeau [4], Smith [5] et Street [11] is rather convincing as for the quality of the model. However, some differences appear.

The model of skier would have a very dissymmetrical technique with long legs contact phase and short arms

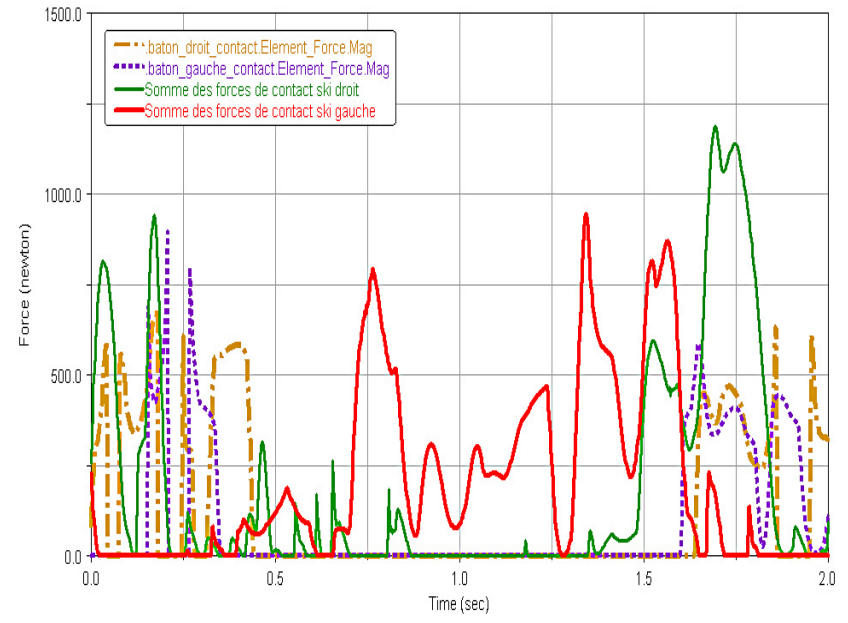

Fig. 21. Poles and skis contact forces from the inverse dynamic simulation.

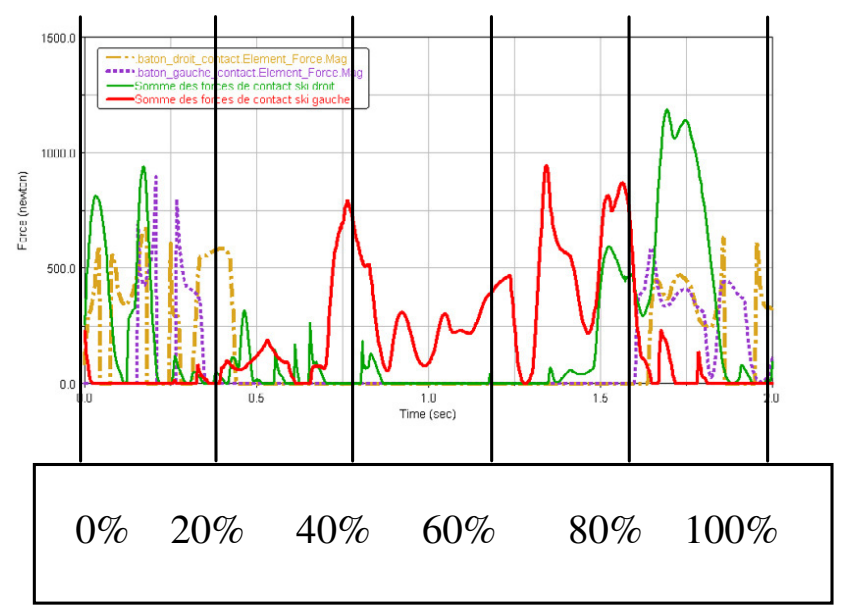

Fig. 22. Poles and the skis contact forces according to the percentage of phase.

contact phase. The gliding phase of the weak leg on this cycle is particularly long.

The poling forces of model are asymmetrical. The skis forces are different. This pattern illustrates common imbalance. The strong side provides an important force and the weak side is for gliding.

The behavior is kinematic and dynamic realistic. So our method is in a good way to simulate with accuracy the skating technique. 


\section{Conclusion}

This article shows above all the difficulty of modelling an environment which interacts on a human model in its movement. It also shows the complexity to build a dynamic modelling from a kinematic modelling. However, results of this study show that this biomechanical approach can directly predict dynamic results based solely on motion kinematic data. Therefore, we collected kinematic, dynamic and also muscular results of this model allowing a fine analysis of the movement [17].

This modelling can also allow comparing technological solutions between them. The industry can be interested.

In perspective, we could make vary external parameters connected to the equipment used and/or to the ski, pole, snow (friction coefficient) or modify internal appropriate parameters for the skier and for his technique, percentage phase of cycle, the edge of ski... The model could be also refined.

\section{References}

[1] F. Rey, J. Corbeau, A. Ferrand, Construction d'un modèle dynamique d'un geste sportif, le pas de patineur, $9^{\text {e }}$ Colloque National AIP PRIMECA, La Plagne, 2005

[2] LIFEMODELER Version BRG. LifeMOD Bio Modeler 2004.g (http://www.lifemodeler.com/)

[3] ADAMS, Using ADAMS/View, Version 12 (http://www. mscsoftware.com/Products/CAE-Tools/Adams .aspx)

[4] B. Bilodeau, M.R. Boulay, B. Roy, Propulsive and gliding phases in four cross-country skiing techniques, Med. Sci. Sports Exerc. 24 (1992) 917-925

[5] G.A. Smith, Biomechanical analysis of cross-country skiing techniques, Med. Sci. Sports Exerc. 24 (1992) 10151022

[6] N. Coulmy, Contribution à l'analyse cinématique et énergétique du pas de patineur en ski de fond, Thèse, Université Joseph Fourier Grenoble I, 2000
[7] P.V. Komi, Ground reaction forces in cross-country skiing, Int. J. Sport Biomech. 3 (1987) 370-381

[8] G.A. Smith, J. McNitt-Gray, R.C. Nelson, Kinematic analysis of alternate stride skating in cross-country skiing, Int. J Sport Biomechanics 4 (1988) 49-58

[9] G.A. Smith, R.C. Nelson, A. Feldmanet, J.L. Rankinen, Analysis of VI skating technique of olympic cross-country skiers, I. J. Sport Biomechanics, 15 (1989) 185-207

[10] G.A. Smith, B.S. Heagy, Kinematic analysis of skating technique of olympic skiers in the men's $50 \mathrm{~km}$ race, J. Appl. Biomech. 10 (1994) 89-90

[11] G.M. Street, R.W. Gregory, Relationship between glide speed and olympic cross-country ski performance, J. Appl. Biomech. 10 (1994) 393-399

[12] R.W. Gregory, S.E. Humphreys, G.M. Street, Kinematic analysis of skating technique of olympic skiers in the women's 30-km race, J. Appl. Biomech. 10 (1994) 382392

[13] A. Ruby, Contribution à la méthodologie de l'analyse de la performance sportive, Thèse, Université Lyon I, 1997

[14] J.P. Verriest, Une méthode simplifiée de simulation du geste du membre supérieur pour mannequin graphique 3D. In : actes du $15^{\mathrm{e}}$ congrès de la société de biomécanique, Cluny, 1990, p. 123-124

[15] M. Tavernier, G. Borsoni, J.P. Verriest, N. Brunel, Méthode de la détermination du centre de masse d'un sportif pour l'analyse du mouvement par images numérisées, Application au ski alpin et au ski de fond, Modèle CGS13. Rapport interne FFS/INREST, 1993

[16] M. Shimbo, Friction on snow of ski soles, unwaked and waxed, Science study of skiing in Japan. Tokyo: The Society of Ski Science, 1971, 101-112

[17] F. Rey, Contribution à la modélisation cinématique et dynamique d'un geste sportif: le pas de patineur, Thèse, Université de Toulouse, 2008 